\title{
Parameterized Study of the Ionospheric Modification Associated With Sun-Aligned Polar Cap Arcs
}

\author{
D. J. CRAIN, J. J. SOJKA, R. W. SCHUNK, AND L. ZHU
}

Center for Atmospheric and Space Sciences, Utah State University, Logan

\begin{abstract}
Sun-aligned (SA) arcs are a prevalent feature of the polar cap ionosphere during northward interplanetary magnetic field conditions. These arcs are, like the auroral arc, a complex electrodynamic system coupling the ionosphere and magnetosphere. The electron precipitation and convection electric field associated with this system modify the polar cap ionospheric plasma distribution. In the past decade, a wealth of observational information has indicated the complexity of these ionospheric modifications, but only a limited number of model studies have been carried out to elucidate the changes associated with SA arcs. In this investigation, an extensive parametric study has been conducted to identify and order these ionospheric modifications. Specifically, we are interested in modeling the local evolution and decay of ionospheric modification due to a source consisting of an idealized, though representative, SA arc structure. The Utah State University time-dependent ionospheric model (TDIM) has been used to define the ionospheric physics and its dependencies on both solar cycle and season. An SA arc model has been developed. This model enables one to systematically vary the key arc parameters; electric field strength, width, electron precipitation energy flux, and electron precipitation characteristic energy. Our findings indicate that the changes associated with the $E$ and $F$ regions are quite different. $F$ region structures have relatively long lifetimes and are significantly affected by transport. This leads to a significant role for cross-arc convection. The $E$ region is much less affected by transport due to short chemical recombination times and thus, $E$ region modifications are confined to the arc structure itself where precipitation and enhanced electric fields are present. Furthermore, the response of the $F$ region to changes in the arc parameters is not linear, and the largest ionospheric modifications occur for intermediate arc widths and electric field strengths. An important indicator of F region modification is the total electron content (TEC) for flux tubes convecting through SA arc structures. The magnitude and variation of the TEC enhancement with various arc parameters can be an important indicator of real processes which are occurring in the arc structure. In addition to TEC we examine the variation of $T_{e}, T_{i}, N_{e}, \Sigma_{\mathrm{P}}$, and $\Sigma_{\mathrm{H}}$ for various arc parameters.
\end{abstract}

\section{INTRODUCTION}

The winter polar cap during northward interplanetary magnetic field (IMF) conditions is often characterized by highly structured electric field and plasma distributions [Berkey et al., 1976; Ismail et al., 1977] often present are Sun-aligned (SA) polar cap arcs which may contribute to this electric field and plasma structuring in an as of yet, unspecified way. Reasons for modeling SA arcs are (1) identifying sources of high-latitude plasma structuring during northward IMF; (2) determining whether a simple SA arc model can reproduce basic features of observed arcs as well as predict possible effects which may be observed by future observations; and (3) correlating specific features of plasma structure $\left(N_{e}\right.$ distribution, TEC enhancement, etc.) to possible causal arc parameters (arc width, cross flow, precipitation, electron flux and characteristic energy, arc electric field, etc.).

Past studies have extensively described the morphology, distribution, and frequency of SA polar cap arcs [Ismail et al., 1977; Gussenhoven, 1982]. These studies have included ground-based optical imagery [Weber and Buchau, 1981; Lassen and Danielson, 1978] and satellite-based optical and particle observations [Hardy et al., 1982; Petersen and Shelly, 1984; Hoffman et al., 1985; Frank et al., 1982, 1986; Akasofu and Tsurutani, 1984; Gorney et al., 1986; Carlson et al., 1984]. It was found that SA arcs were observed most frequently

\section{Copyright 1993 by the American Geophysical Union.}

Paper number 92JA02668.

0148-0227/93/92JA-02668\$05.00 during geomagnetic quiet times characterized by northward IMF [Akasofu, 1972; Berkey et al., 1976; Berkey and Kamide, 1976; Gussenhoven, 1982].

The characteristics of SA polar cap arcs span a wide range of spatial sizes and optical brightness. The most prominent of these arcs may extend several thousand kilometers across the polar cap and have a width of several hundred kilometers. The brightest of these transpolar arcs are sometimes known as "theta" aurora [Frank et al., 1982, 1986]. Often, a theta aurora may be composed of several discrete arcs parallel to each other in the noon-midnight direction [Akasofu and Tsurutani, 1984]. There is also evidence that many polar cap auroras may be a conjugate phenomena appearing in both hemispheres [Obara et al., 1988; Mizera et al., 1987] and that they may be more common than previously thought, as at times they may be subvisual [Weber and Buchau, 1981]. Other SA polar cap arcs may extend poleward from the midnight sector of the auroral oval or exist discretely within the polar cap with no obvious connection to the auroral oval [Akasofu and Roederer, 1983; Hoffman et al., 1985].

There remains much debate as to the mechanism of SA arc formation, especially as to the specific magnetospheric topology and plasma population which produces the observed arcs. There does, however, seem to be some consensus on the general features of SA polar cap arcs. They are usually characterized by a region of large-scale shear flow in the plasma convection consistent with a region of $\nabla \cdot \mathbf{E}<0$, where $\mathbf{E}$ is the horizontal convection electric field [Weber et al., 1989]. There is also a weak upward current associated with precipitation of particles of energy less than a few $\mathrm{keV}$ near the 
region of $\nabla \cdot \mathbf{E}<0$. Horizontal electric fields within such arcs may be as high as $100 \mathrm{mV} / \mathrm{m}$, but are usually much lower.

Coordinated rocket, satellite, and radar campaigns have attempted to comprehensively describe the ionospheric signatures of such arcs [Niciejewski et al., 1989; Weber et al., 1989]. Basu et al. [1988, 1990] have examined the plasma structuring in the polar cap associated with SA arcs through use of GPS and polar beacon satellites which measure TEC and scintillation signatures of the plasma density gradient and shear flow associated with SA arcs. They observed a solar cycle dependence upon the TEC enhancement associated with SA arcs. A generalized SA arc based on a compilation of these campaigns and other radar and satellite observations has been recently developed by Valladares and Carlson [1991]. While the optical, electrodynamic, and particle precipitation characteristics of polar cap arcs have been extensively described, no systematic study of the effect of typical SA polar cap arcs upon the high-latitude ionosphere has been done. In a few specific SA arc observations the ionospheric modifications have been noted. Theoretically, the extent of these modifications will depend upon the specific convection electric fields and particle precipitation in a complex manner. Sojka and Schunk [1987], modeled the polar ionosphere for different northward IMF convection patterns, and later Sojka and Schunk [1988] modeled the polar ionosphere's response to small-scale (SA arc scale) convection electric field structures. These studies focused on the effects associated with the convection and did not include those effects associated with the particle precipitation. The effects due to particle precipitation were studied by Schunk and Sojka [1987], but this study did not include SA arc electric field structures. These plasma enhancements were associated with southward IMF polar cap patches. In addition, both of these studies were essentially global in that they examined the modification of the entire polar ionosphere due to various global convection patterns associated with northward IMF.

In this paper we will be primarily concerned with the local ionospheric modification that is due to a generalized steady state arc structure. The SA arc structure is constructed to be locally consistent with observed arcs. We do not attempt to address questions of magnetospheric topology which produce the shear flow and particle precipitation associated with SA polar cap arcs. (Nor do we address the large-scale Birkeland current system associated with positive $B_{z}$.) These local modifications are nevertheless important as they contribute to the overall plasma structuring of the entire polar cap, especially during northward IMF conditions. An ability to better understand these local processes may lead to a better capability in modeling the highly structured polar cap [Basu et al., 1990].

For a representative set of SA arc parameters which includes both convection and precipitation, we will concentrate on the modification by SA polar cap arcs upon the $F$ region electron density and the height integrated conductivity. Our findings will be summarized in a way that parameterizes the effect of specific arc parameters upon a particular ionospheric parameter.

\section{ARC MODEL}

The ionosphere model used in this study is the Utah State University time-dependent ionospheric model (TDIM). This is a first principle ionospheric model which self-consistently solves the continuity, momentum, and energy equations for a multi-ion plasma $\left(\mathrm{NO}^{+}, \mathrm{O}_{2}^{+}, \mathrm{N}_{2}^{+}, \mathrm{O}^{+}, \mathrm{N}^{+}\right.$, and $\mathrm{He}^{+}$) along an ionospheric flux tube. The TDIM was developed over a 2 decade period by Schunk and coworkers. Its theoretical development is described by Schunk [1988], while its comparison with observations by Sojka [1989]. In addition to the ionosphere model [Sojka and Schunk, 1988], a SA arc model was developed. This SA arc model defines a structure in the convection and precipitation inputs that is representative of a SA arc.

The arc model itself was constructed to be simple and yet be representative of a wide range of SA polar cap arcs. The location and orientation of the arc structure is easily varied and may be situated anywhere in the polar cap. For this study, we orientate the model arc on the noon-midnight median passing through the magnetic pole. The relevant variable parameters of the arc are: the width in the dawn-dusk direction; the distribution of the electric potential; the characteristic energy $(\mathrm{keV})$; and energy flux (ergs $\mathrm{cm}^{-2} \mathrm{~s}^{-1}$ ) of the particle precipitation. A background convection pattern is required. This large-scale convection transports plasma into the local $\mathrm{SA}$ arc environment. In general, no unique northward IMF convection pattern is available. For the purposes of this study where relative plasma effects in the SA arc region are sought, we only require that the large-scale convection brings representative plasma into the SA arc region. Given this minimal requirement we have adopted a plasma flux tube trajectory from a $B_{z} \sim 0$ and strong $B_{y}$ pattern to convect plasma into the SA arc region. The results of this study are not sensitive to this choice.

Once in the SA arc region, the plasma flux tube motion is determined by a superposition of the SA arc and background electric fields. This raises yet another unresolved magnetospheric issue; namely, SA arcs occur at/or near convection shears, but is there a cross arc drift that carries plasma through the arc? Our results will show that observations of the plasma on and near arcs can put strong limits on this motion. (In this study we vary the cross arc drift by changing the background convection.) Since the plasma remains in the arc for time intervals of less than about 1 hour, we have chosen to make the background convection and SA arc model time independent. Temporal variations would be the topic of future work. In this study, the length of the SA arc and its exact orientation relative to the Sun-aligned direction are not studied since we are following only one flux tube as it crosses the SA arc region. If plasma flux tubes do not drift across the SA arc (or the arc moves relative to the plasma) the flux tube would remain in the arc for long periods of time since SA arcs are observed to be stable for tens of minutes to hours [Valladares and Carlson, 1991; C. E. Valladares et al., The IMF dependence of stable Sun-aligned polar cap arcs, submitted to Journal of Geophysical Research, 1993]. In this case, the plasma would drift but stay inside the arc and densities would increase. Our results predict that for such "stagnant" conditions well-defined plasma signatures should be observed. (By varying the background electric field we can simulate this extreme condition.)

The dawn-dusk distribution of these parameters are summarized in Figure 1 for an arc width of $200 \mathrm{~km}$ traversing the $\mathrm{SA}$ arc in the midnight sector of the polar cap. Figure $1 a$ shows the arc potential distribution which has a $2.2-\mathrm{kV}$ potential well; Figure $1 b$ shows the corresponding electric fields, and Figure $1 c$ shows the electron precipitation distribution. In order to keep the arc simple, the potential well 


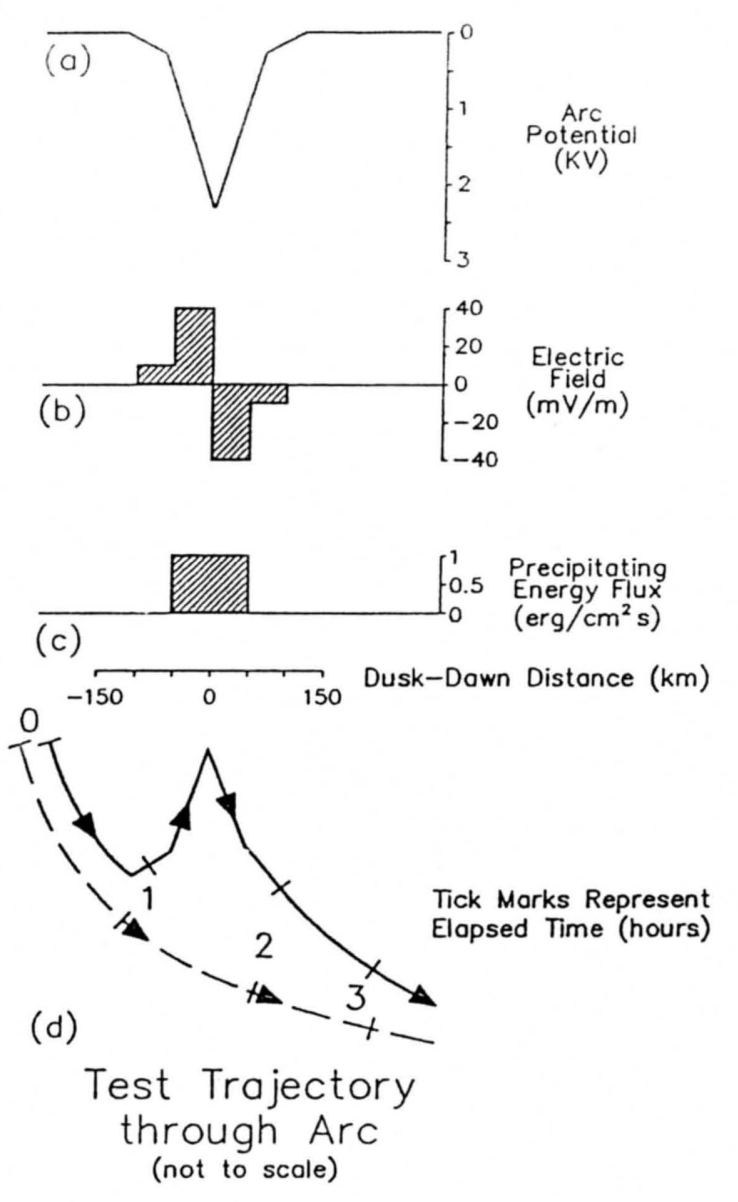

Fig. 1. Dawn-dusk distribution of the prototype SA polar cap arc parameters. The horizontal axis represents spatial size in kilometers: (a) Potential structure within the arc; $(b)$ arc electric field across arc structure dusk-dawn direction is positive; $(c)$ energy flux of the precipitating electrons; and $(d)$ general convection trajectory of a flux tube convecting through arc structure.

was chosen to be a ramped vee for which discrete electric field values within the arc could be determined. The potential well was ramped so that for a particular arc width, the maximum electric field values and precipitation were centered within the center portion of the arc potential structure. For the arc in Figure 1, the width of the potential well was $200 \mathrm{~km}$ wide. Within this well was a $100 \mathrm{~km}$ wide precipitation region and a $100 \mathrm{~km}$ wide region where the electric field was maximized. The effect of the electric field upon the convection trajectory of a particular flux tube is shown schematically in Figure 1d. The dashed line indicates the background convection without the arc structure. The solid line indicates the convection trajectory for the superposition of the background and arc electric field. The tic marks along the solid line indicate elapsed time along the trajectory.

This model of a SA polar cap arc is intended to be reasonably consistent with the gross nature of observed SA polar cap arcs. Namely, the arc occurs in a region of $\nabla \cdot \mathbf{E}<0$, the arc is aligned in a noon-midnight direction, the length of the arc is much greater than its width and the precipitation structure is centered near a shear in flow (or electric field).

In addition to the model arc parameters being varied, the effect of both season and solar cycle level was considered. These latter two geophysical conditions were defined as inputs to the TDIM. The TDIM was otherwise configured as described by Sojka and Schunk [1988].

\section{RESULTS}

For simplicity and to aid in the interpretation of the effect of particular arc parameters upon the ionosphere we introduce a prototype arc. This arc structure has a width of $200 \mathrm{~km}$ and a maximum arc electric field of $40 \mathrm{mV} / \mathrm{m}$. The precipitation is a Maxwellian electron beam of $0.5-\mathrm{keV}$ characteristic energy and total energy flux of $1 \mathrm{erg} \mathrm{cm}^{-2} \mathrm{~s}^{-1}$. The relevant ionospheric conditions are for winter solar minimum $\left(F_{10.7}=70\right)$. The location of the arc traversal is in the midnight sector (MLT) of the polar cap. This prototype arc will be used in the following parameter study. In each of the following cases, one parameter of the prototype arc or ionospheric condition is varied with al other parameters remaining the same.

An ionospheric pseudo cross section of this arc simulation is shown in Figure 2. The horizontal axis represents elapsed time in hours for a flux tube convecting upon a particular polar cap trajectory. Since the flow across the arc is relatively constant, the horizontal axis also represents horizontal distance across the arc. The arc location is identified in Figure $2 c$ by the two vertical dashed lines. Figure $2 a$ shows the variation of total electron content (TEC) across the arc structure. The solid line represents the time (spatial) variation of TEC for a flux tube convecting across the arc structure which has a precipitation structure imbedded in it as described previously (see Figure 1).

Figure $2 b$ represents the variation of $\Sigma_{H}$ and $\Sigma_{P}$ across the arc. The heavy line represents the variation of $\Sigma_{P}$ across the arc and the thin line represents $\Sigma_{H}$. Figure $2 c$ is a contour plot of the electron density between 100 and $800 \mathrm{~km}$ across the arc. The maximum contour corresponds to a density of $10^{5} \mathrm{~cm}^{-3}$, and the minimum contour is $10^{1.5} \mathrm{~cm}^{-3}$.

From Figure 2 we can readily see some of the gross effects of a SA polar cap arc on the ionosphere. The enhanced $\Sigma_{H}$ and $\Sigma_{P}$ occurs within the precipitation region of the arc structure and corresponds to the enhanced $E$ region densities due to the electron precipitation. The TEC increases across the arc and remains elevated outside the arc corresponding to the enhanced plasma densities at $F$ region altitudes observed after the flux tube crosses the arc (see Figure 2c). For this study we will use TEC as an indicator of the relative $F$ region enhancement and $\Sigma_{H}$ and $\Sigma_{P}$ as indicators of the relative $E$ region enhancement in addition to density contour plots.

An examination of the contour plot of $N_{e}$ in Figure $2 c$ allows us to make some qualitative observations of the effect of arc structures upon the ambient ionosphere. The most dramatic effect is seen on the edge of the arc as the flux tube convects into it. The rapid rise in $T_{e}$ due to the topside heat flux of the precipitating electrons and, to a lesser extent, upon Joule heating due to $\mathbf{E} \times \mathbf{B}$ convection produce an immediate increase in plasma scale height within the arc structure. This is seen as an increase in $N_{e}$ at very high altitudes. As the flux tube moves out of the enhanced $T_{e}$ and $T_{i}$ regions, the scale heights decrease resulting in a sharp decrease in $N_{e}$ at high altitudes. Near the $F$ peak, the combination of enhanced production due to electron precipitation, vertical transport, and thermal diffusion combine to produce an increasing electron concentration across the arc. This produces an enhanced $F$ region as the flux tube moves outside of the arc structure that may be maintained for many hours. This enhancement and maintenance of the $F$ region density serves to enhance the TEC of the flux tube.

In the $E$ region, the primary effect of the arc structure is to produce enhanced ionization due to the electron precipitation. 

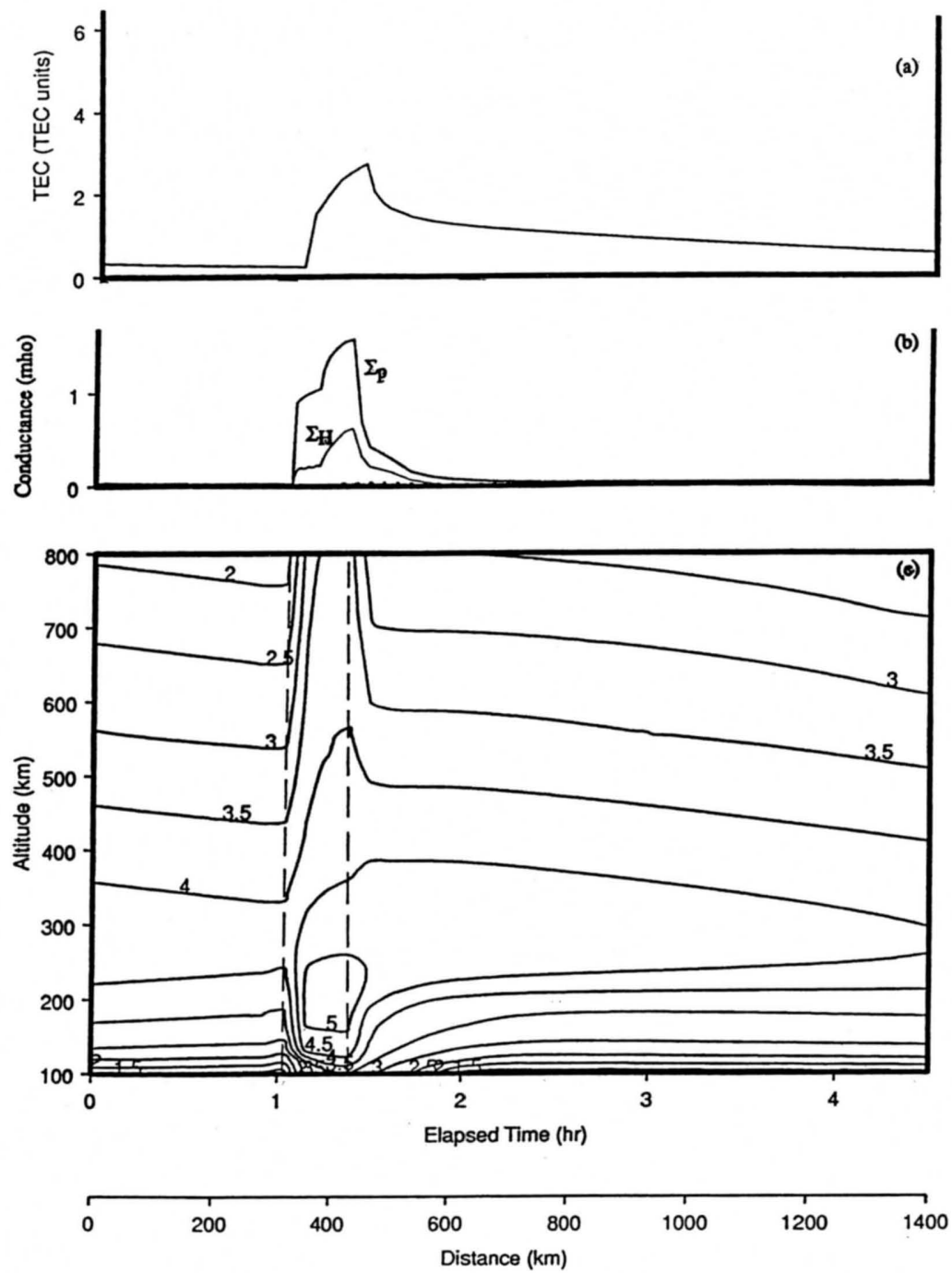

Fig. 2. Ionospheric cross section of the prototype SA polar cap arc. (a) The variation of TEC of a flux tube traversing the arc structure from the left ( 1 TEC unit $=1 \times 10^{6}$ electrons $\left./ \mathrm{m}^{2}\right),(b)$ the variation of $\Sigma_{P}$ and $\Sigma_{H}$ across the arc structure; and (c) contours of the computed $N_{e}$ distribution across the arc structure. The relevant arc conditions are winter solar minimum $\left(F_{10.7}\right.$ $=70$ ), arc electric field $=40 \mathrm{mV} / \mathrm{m}$, arc width $=200 \mathrm{~km}$, characteristic energy of precipitating electrons is $0.5 \mathrm{keV}$ and the energy flux of the precipitation is $1 \mathrm{erg} \mathrm{cm}^{-2} \mathrm{~s}^{-1}$. A distance from the arc (dawn-dusk direction) is shown below the flux tube transit time axis.

This enhancement is localized within the arc structure and quickly disappears as the flux tube moves out of the arc region. This is also reflected in the variation of $\Sigma_{H}$ and $\Sigma_{P}$ across the arc. The integrated conductivities are most enhanced within the precipitation region. If the $F$ region is significantly enhanced, there may also be a significant contribution to the total integrated conductivity from altitudes above $150 \mathrm{~km}$. The general features of the prototype arc correlate with the composite arc constructed by Valladares and Carlson [1991] and displayed in Figure 14 of that paper.

\subsection{Temperature and Transport Effects}

Within the modeled SA polar cap arcs there are two sources of temperature enhancement. The first source is the $T_{e}$ enhancement due to the topside heat flux of the precipitating electrons. In this work we assume that the heat flux is proportional to the energy flux of the electrons following the work of Schunk et al. [1986]. This electron heating couples to the ions self-consistently at all altitudes and to the neutral gas at the lowest altitudes. The most prominent effect of the elevated $T_{e}$ within the arc is to increase the topside scale height of the plasma. In all cases, this serves to increase the $\mathrm{O}^{+}$ density on the topside of the $F$ region. Figure 3 shows vertical profiles of $T_{e}$ for the prototype arc for three different precipitation fluxes $\left(0.1,1\right.$, and 5 ergs $\left.\mathrm{cm}^{-2} \mathrm{~s}^{-1}\right)$. The other parameters are the same as those used in Figure 2. These profiles are located at the center of the arc.

The second source of temperature enhancement is by Joule heating due to the component of the electric field perpendicular to the geomagnetic field line. The enhancement in the ion temperature maximizes at low altitudes and increases rapidly for electric fields greater than $40 \mathrm{mV} / \mathrm{m}$ [Sojka and Schunk, 1983]. Most of the effects of $T_{i}$ enhancements upon the evolution of the daytime and nighttime plasma distribution (due to electric fields) have been described in previous work [Schunk et al., 1975, 1976, 1980]. 


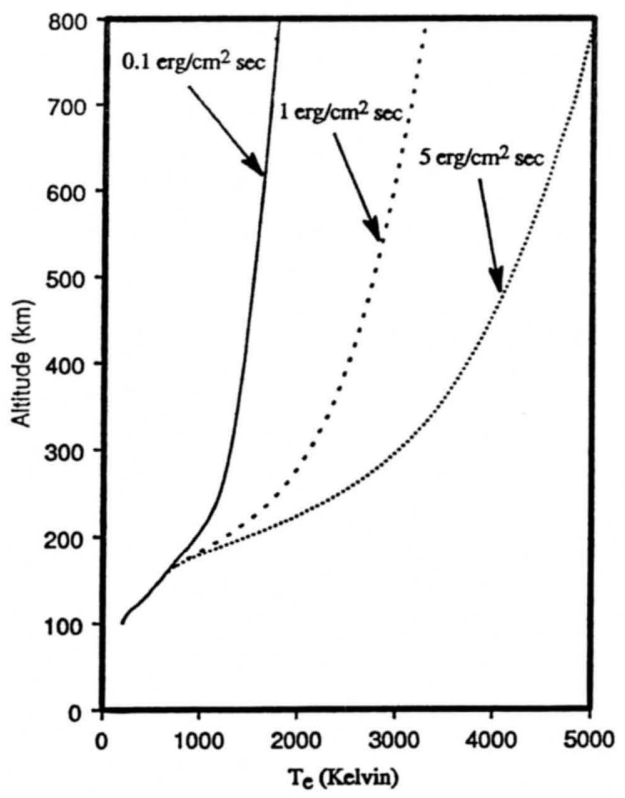

Fig. 3. Plot of the vertical $T_{e}$ profiles within the prototype arc for energy fluxes of 0.1 (solid line), 1.0 (dashed line), and 5.0 (dotted line) ergs $\mathrm{cm}^{-}$ $2 \mathrm{~s}^{-1}$.

These heated ions couple to the electrons and serve to generally enhance the molecular ion concentrations at all altitudes due to the following reaction rates being strongly temperature dependent [Schunk et al., 1975]:

$$
\begin{gathered}
\left(\mathrm{O}^{+}+\mathrm{N}_{2} \rightarrow \mathrm{NO}^{+}+\mathrm{N}\right) \\
\left(\mathrm{O}^{+}+\mathrm{O}_{2} \rightarrow \mathrm{O}_{2}^{+}+\mathrm{O}\right)
\end{gathered}
$$

Figure 4 shows the $T_{i}$ profile within the prototype arc for $10,40,60,80$, and $100-\mathrm{mV} / \mathrm{m}$ electric fields. The other parameters are the same as those used in Figure 2. These profiles show how the bottomside ion temperature becomes enhanced for electric fields greater than $60 \mathrm{mV} / \mathrm{m}$.

For the stronger zonal electric field cases, there is also a significant transport effect associated with the vertical component of the $\mathbf{E} \times \mathbf{B}$ convection. Therefore, within the arc there may exist not only a shear flow in the horizontal direction (sunward/antisunward) but in the vertical direction as well. The sign of the vertical drift depends upon dip angle and is minimized at higher magnetic latitudes. In the $E$ region the plasma is inhibited from moving in the $\mathbf{E} \times \mathbf{B}$ direction as the ion neutral collision frequency $\left(\gamma_{\text {in }}\right)$ increases with decreasing altitude. Hence this effect is only an $F$ region mechanism which raises or lowers the layer.

\subsection{Solar Cycle Dependence}

The first parameter which will be varied is the $F_{10.7}$ solar flux. Figure 5 shows a contour plot of $N_{e}$ (bottom panel), $\Sigma_{H}$ and $\Sigma_{P}$ (middle panel), and TEC for winter solar maximum $\left(F_{10.7}=210\right)$ ionospheric conditions. The other parameters are the same as those used for Figure 2. Please note the case without the precipitation is not shown in the top panel of Figure 2.
For solar maximum, the relative enhancement is much less at high altitudes ( $F$ region) but the absolute enhancement is larger at all altitudes compared to solar minimum (compare Figures 2 and 5). The $F$ region enhancement occurs at higher altitudes due to the larger-scale height of the neutral atmosphere which has raised $h_{m} F_{2}$ from below $300 \mathrm{~km}$ (solar minimum) to about $350 \mathrm{~km}$ (solar maximum). This also moves upward the region of enhanced ionization due to the soft $(0.5$ $\mathrm{keV}$ ) precipitation. This raising of the aurorally produced ionization leads to a slight enhancement of $\Sigma_{P}$ and hence a small $\sim 10 \%$ change in the $\Sigma_{\mathrm{P}} / \Sigma_{\mathrm{H}}$ ratio. In the topside ionosphere, the enhanced topside scale height is readily evident, i.e., few contour lines during solar maximum. The solar maximum background densities for this trajectory are almost an order of magnitude higher than at solar minimum. These higher densities are now on the same order as the density enhancement associated with the $1 \mathrm{erg} \mathrm{cm}^{-2} \mathrm{~s}^{-1}$ arc. Basu et al. [1990] have observed that the relative TEC enhancement associated with SA polar cap arcs varies with solar cycle. They observed typical $\triangle$ TECs of $\sim 5 \times 10^{16}$ electrons $/ \mathrm{m}^{2}$ for solar maximum conditions and $\sim 2 \times 10^{16}$ electrons $/ \mathrm{m}^{2}$ for solar minimum conditions. We have examined the $\triangle T E C$ associated with the modeled prototype arc for solar minimum and maximum conditions. Figure 6 shows the $\triangle T E C$ enhancements versus characteristic energy of the precipitating electrons for solar minimum and maximum. The TEC enhancement is heavily dependent on characteristic energy of the precipitation but does show the general trend observed by Basu et al. [1990].

\subsection{Electric Field Strength Comparison}

Next, we examine the effect of the arc electric field strength on the ionosphere. Again, the arc structure described previously for Figure 2 is used. The arc electric field is varied in $10 \mathrm{mV} / \mathrm{m}$ increments from 10 to $100 \mathrm{mV} / \mathrm{m}$. Figures $7 a$ and $7 b$ show the $N_{e}$ contours for the 10 and $100 \mathrm{mV} / \mathrm{m}$ cases, respectively, while Figure 2 is for $40 \mathrm{mV} / \mathrm{m}$.

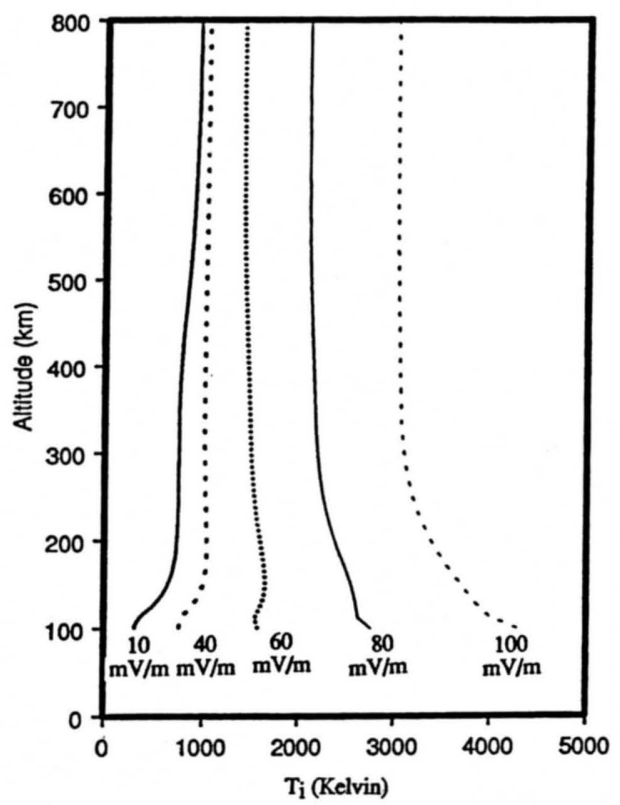

Fig. 4. Vertical profiles of $T_{i}$ within the prototype arc for arc electric fields of $10,40,60,80$, and $100 \mathrm{mV} / \mathrm{m}$. 

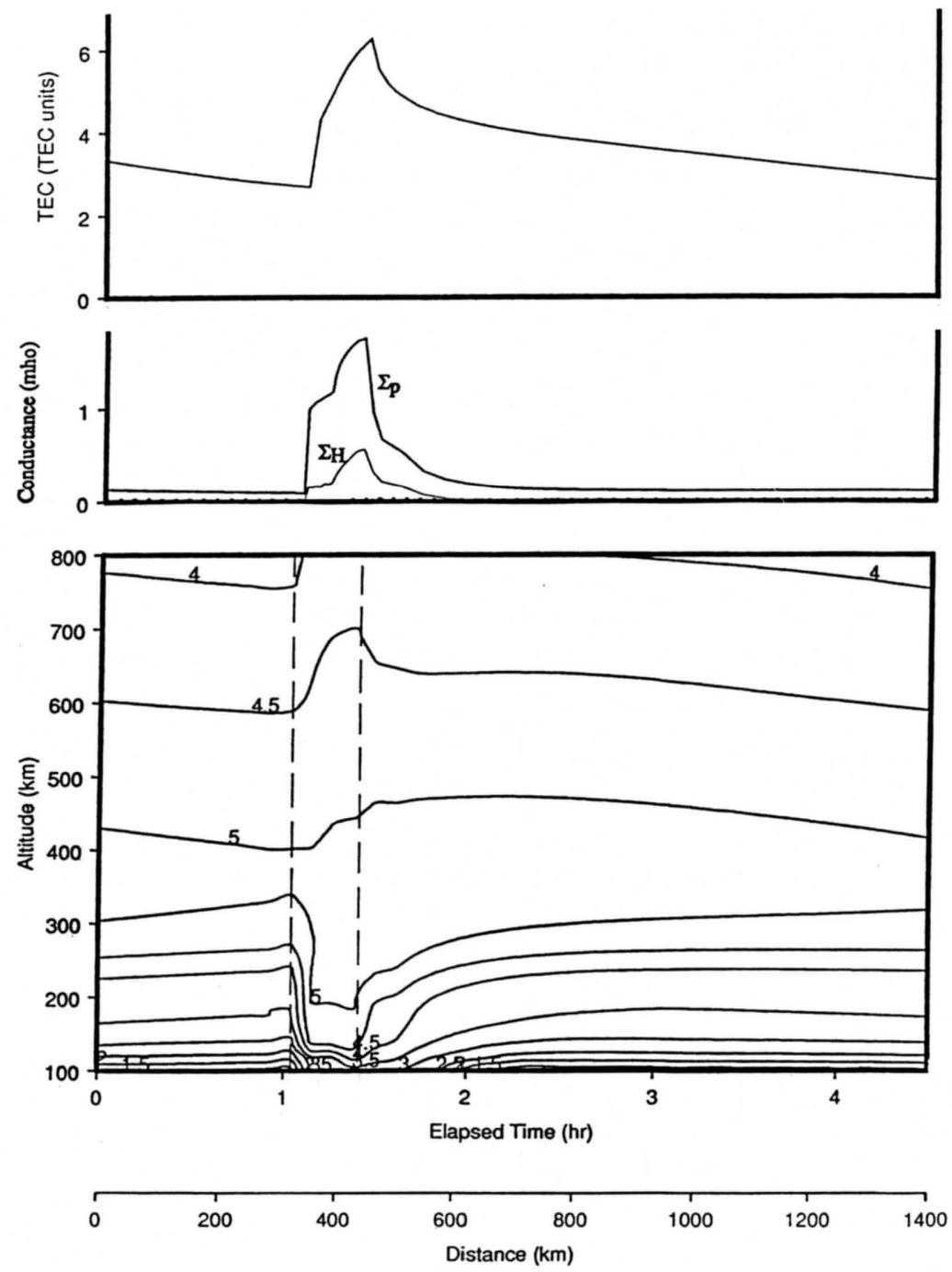

Fig. 5. Same as Figure 2 except for winter solar maximum conditions $\left(F_{10.7}=210\right)$.

In general, the plasma enhancement due to the SA arc increases for increasing arc electric field between 10 and 40 $\mathrm{mV} / \mathrm{m}$. As the arc electric field increases above $40 \mathrm{mV} / \mathrm{m}$, the plasma enhancement becomes smaller, especially in the $F$ region. For this particular arc structure, the plasma enhancement of the ionosphere maximizes for an arc electric field near $40 \mathrm{mV} / \mathrm{m}$. The competing processes, enhanced production, transport, and thermal diffusion produce a maximum enhancement. As the electric field increases above $40 \mathrm{mV} / \mathrm{m}$, more of the enhanced ionization is converted to molecular ions due to the temperature dependent reactions in equations (1) and (2). Therefore the overall density is lower and decays more rapidly when the flux tube leaves the arc due to the faster recombination times for the molecular ions. Figure 8 quantifies the magnitude of the electric field dependence by showing the $N_{m} F_{2}$ (solid line) inside the arc and the $N_{m} F_{2}$ (dotted line) $\sim 20 \mathrm{~min}$ after leaving the arc. The $N_{m} F_{2}$ versus electric field strength trend is readily seen, as is the peak around $40 \mathrm{mV} / \mathrm{m}$.

In the $E$ region, the variation of $\Sigma_{H}$ and $\Sigma_{P}$ across the arc is about the same for all electric field strengths. For very large fields, there may be important sources of enhancement which are beyond the capability of this work to examine.
Specifically, there may be significant effects due to realistic $E$ region transport, wave particle heating, and electrodynamics which we do not address here.

\subsection{Characteristic Energy Comparison}

Previously, we examined the effect of characteristic energy versus $\triangle T E C$ in Figure 6. TECs increased significantly for low characteristic energy corresponding to enhanced $F$ region production. This is also evident if we examine the ratio of $\Sigma_{P} / \Sigma_{H}$ versus characteristic energy. Figure 9 shows the variation of the $\Sigma_{P} / \Sigma_{H}$ ratio versus characteristic energy for solar minimum and solar maximum conditions. These results are comparable to values previously calculated by Vickery et al. [1981] and Rasmussen and Schunk [1988]. The effect of increasing the characteristic energy is to lower the altitude of maximum production. This serves to decrease the TEC as seen in Figure 6 and to increase the conductances. The $\Sigma_{P}$ shows a small increase with increasing characteristic energy, while $\Sigma_{H}$ is dramatically increased (a factor of 12 from the 0.5 to $5 \mathrm{keV}$ cases). The net result upon the $\Sigma_{\mathrm{P}} / \Sigma_{\mathrm{H}}$ ratio is that it decreases from a value of $\sim 3.0$ to $\sim 0.5$. This may lead to important 


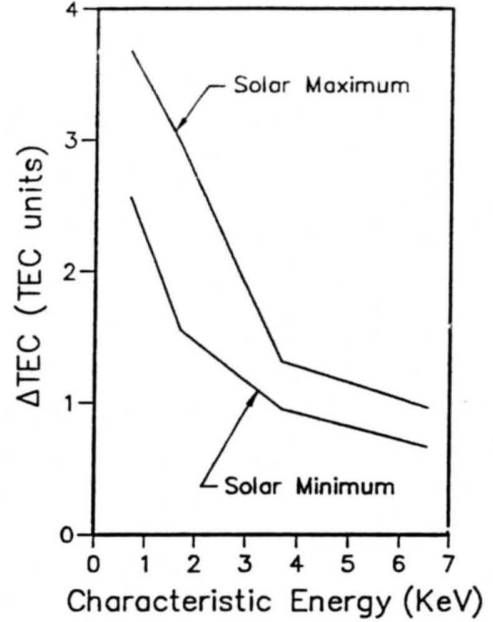

Fig. 6. Same as Figure 2 except for summer solar minimum conditions.

differences in the electrodynamics and current flow associated with the arc for differing precipitating particle characteristic energies. The $F$ region enhancements decrease with increasing characteristic energy as indicated by the TEC. Specifically, the $N_{m} F_{2}$ enhancement in the arc decreases from a factor of 10 at $0.5 \mathrm{keV}$ to a factor of 4 at $5 \mathrm{keV}$. These factors, since they are relative, will depend upon ionospheric conditions and the other arc parameters.

\subsection{Arc Width Comparison}

The effect of arc width upon the plasma density is represented in Figure 10. Figure 10a shows a contour plot of $N_{e}$ for a $100-\mathrm{km}$-wide prototype arc. Comparing Figure $10 a$ to Figure $2 c$ we can see that the $100-\mathrm{km}$-wide arc produces less of an $F$ region enhancement than the $200-\mathrm{km}$-wide arc. The enhancement below $200 \mathrm{~km}$ is roughly equal for both cases, differing only by the spatial size of the precipitating region. In Figure $10 b$ we show the $N_{e}$ contour for a $400-\mathrm{km}$-wide arc. When compared to Figure $2 c$, there is only a very moderate enhancement in the $F$ region density for the larger arc. Again, the enhancement below $200 \mathrm{~km}$ is about the same except for the width of the precipitation region.

For the simulations performed in this study the degree of $F$ region enhancement is approximately linear for increasing arc width below $200 \mathrm{~km}$. As the width of the arc exceeds $200 \mathrm{~km}$, the degree of $F$ region enhancement saturates and there is little further enhancement with increasing width.

The arc width may also be viewed as the time spent in the arc ionization region. Hence the wider arcs correspond to longer times in the ionization region which gives the flux tube $F$ region time to reach "steady state" for the given auroral ionization. For the Figure 2 and 10 arc scenarios this is approximately reached for the $200-\mathrm{km}$-wide arc. This arc width/time in our discussion also depends on the background electric field strength. For a flux tube that moves quickly (large electric field) across a wide arc the degree of enhancement may be less than for a flux tube which moves slowly across a much narrower arc. In addition, this does not address the issue of the arc structure itself producing a significant cross arc flow component due to self-consistent magnetospheric-ionosphere electrodynamic coupling [Zhu et al., 1992]. The extreme case of very little cross arc drift is discussed below.

\subsection{Stagnation}

In the limiting case where the plasma flow across the arc is zero, the flux tube can be thought of as "stagnating" within the precipitation of the SA arc structure. If this occurs, the flux tube may spend a much longer amount of time within the region of enhanced precipitation and sunward/antisunward convection. To examine this in a simple way, we have again used the prototype arc structure. After the flux tube convects into the arc structure the cross drift is set to zero and the flux tube only

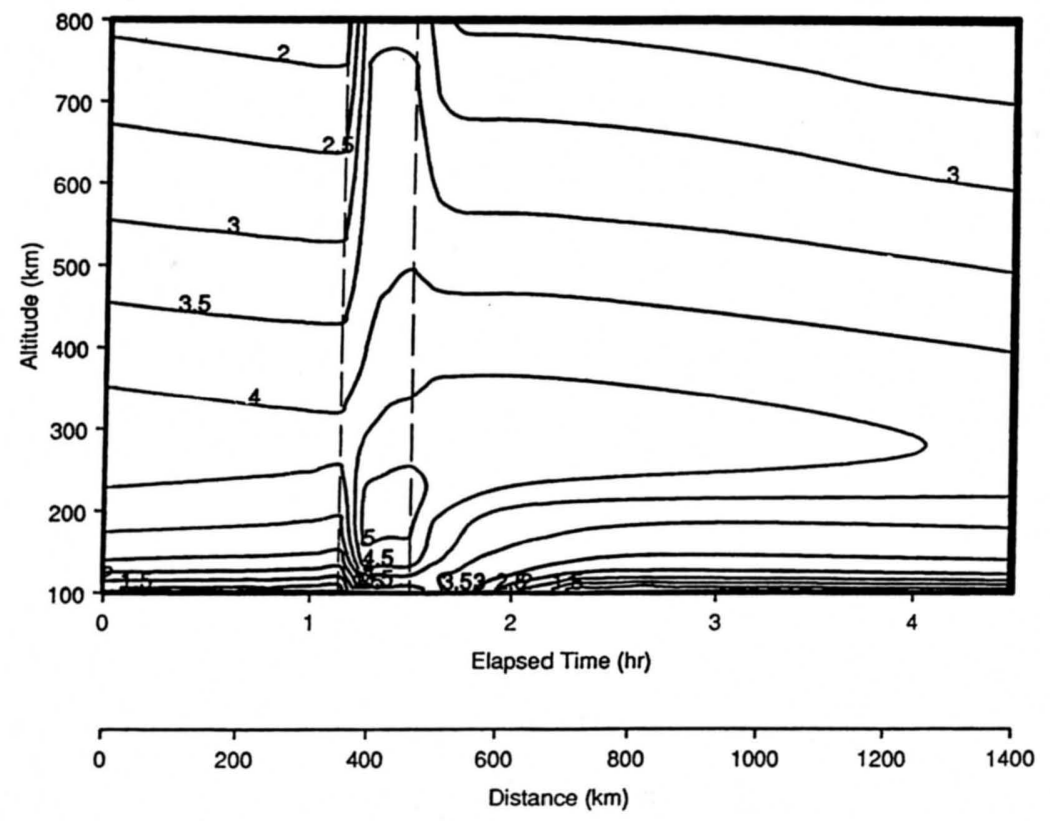

Fig. 7a. $N_{e}$ contour plot for the prototype arc with an arc electric field of $10 \mathrm{mV} / \mathrm{m}$. The relevant arc conditions are arc width equal to $200 \mathrm{~km}$, characteristic energy of precipitating electrons is $0.5 \mathrm{keV}$, and the energy flux of the precipitation is $1 \mathrm{erg} \mathrm{cm}^{-}$
$\mathrm{s}^{-1}$. 


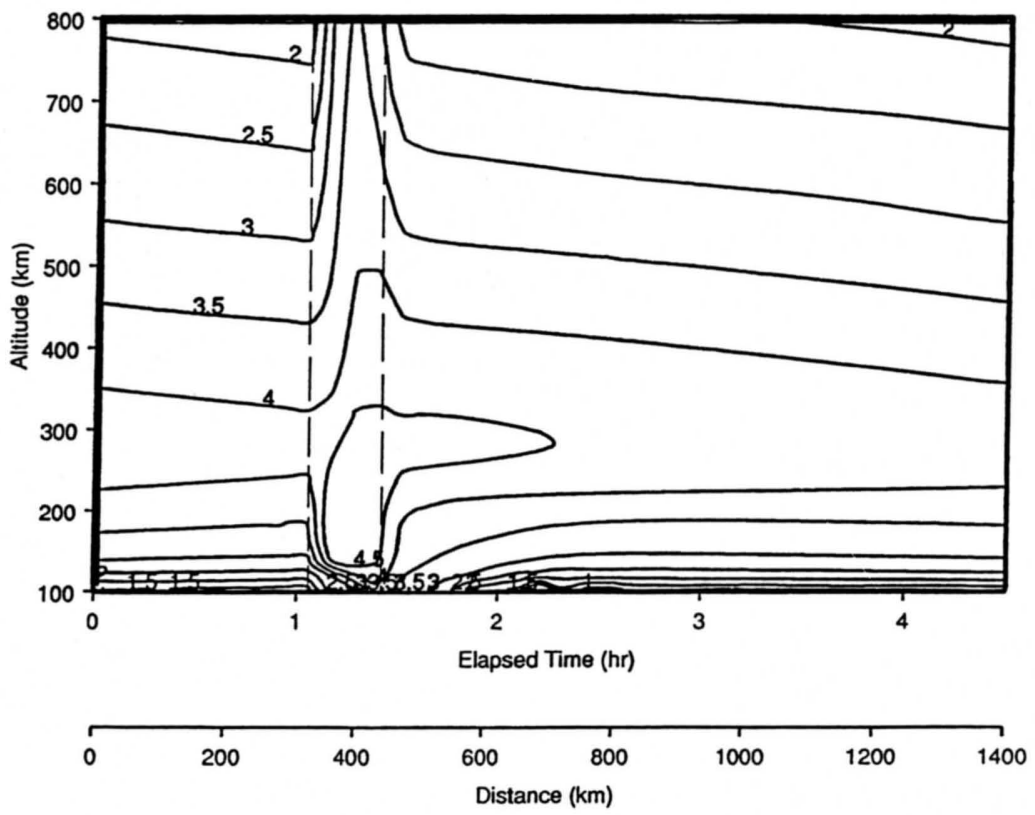

Fig. $7 b . N_{e}$ contour plot for the prototype arc with an arc electric field of $100 \mathrm{mV} / \mathrm{m}$. The relevant arc conditions are arc width equal to $200 \mathrm{~km}$, characteristic energy of precipitating electrons is $0.5 \mathrm{keV}$, and the energy flux of the precipitation is $1 \mathrm{erg} \mathrm{cm}^{-}$ $2 \mathrm{~s}^{-1}$.

moves in the sunward or antisunward direction. Figure 11 shows the TEC enhancement associated with such a "stagnated" flux tube. The top curve is for solar maximum and the bottom curve is for solar minimum. If the flux tube stays within the precipitation region for a significant amount of time $(\sim 1$ hour), the TEC enhancement can be very significant, of the order of $\sim 6 \times 10^{16}$ electrons $/ \mathrm{m}^{2}$ which is much larger than observed values [Basu et al., 1990]. In order to produce a more reasonable TEC enhancement in a flux tube which has stagnated within the SA arc we must then either decrease the flux or increase the characteristic energy of the precipitation.

Figure 12 shows the TEC enhancement as in Figure 11 associated with the prototype arc but with a flux of 0.1 ergs $/ \mathrm{cm}^{2} \mathrm{~s}$. The TEC enhancement after approximately 1 hour is now less than $\sim 2 \times 10^{16}$ electrons $/ \mathrm{m}^{2}$. These monotonically increasing TEC trends are distinctly different from the $N_{m} F_{2}$ trends found in section 3.6. $N_{m} F_{2}$

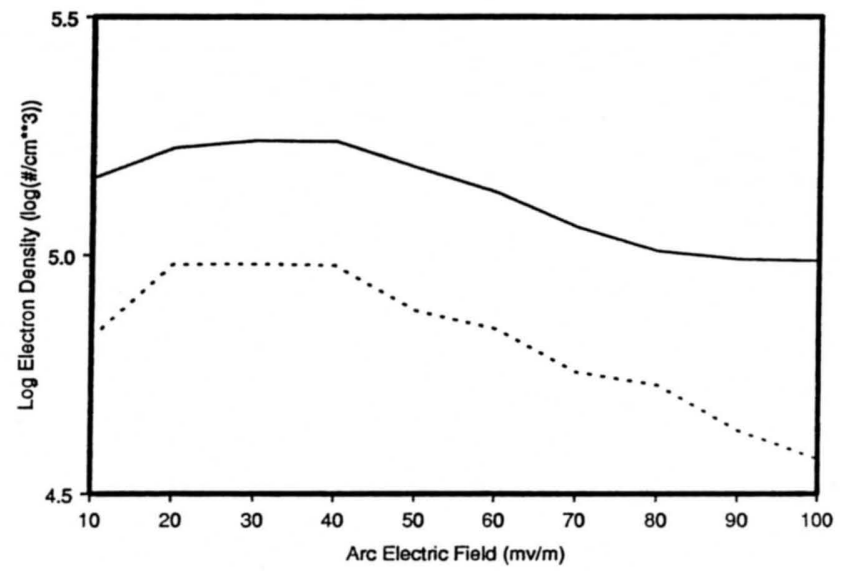

Fig. 8. $N_{m} F_{2}$ versus arc electric field inside the precipitation region (solid line) and $\sim 20 \mathrm{~min}$ after leaving the precipitation region. asymptotically approaches a limit. This limit takes longer than say the $E$ region chemistry time constant of seconds; however, it is much less than an hour. This contrasting $N_{m} F_{2}$ TEC behavior is potentially a useful tool to establish how long plasma flux tubes have been within an arc. However, as Figures $2,10,11$, and 12 indicate, arc parameters such as characteristic energy would be needed in addition to $N_{m} F_{2}$ and TEC observations.

\subsection{Flux Comparison}

Finally, we will examine the effect of varying the precipitating electron flux in the arc. Figure $13 a$ shows the TEC, variation across the prototype arc for energy fluxes of 0.1 and $5 \mathrm{erg} \mathrm{cm}^{-2} \mathrm{~s}^{-1}$. Figure $13 b$ shows the variation of $\Sigma_{H}$ and $\Sigma_{P}$ across the arc for energy fluxes of 0.1 and $5 \mathrm{ergs} \mathrm{cm}^{-2} \mathrm{~s}^{-1}$.

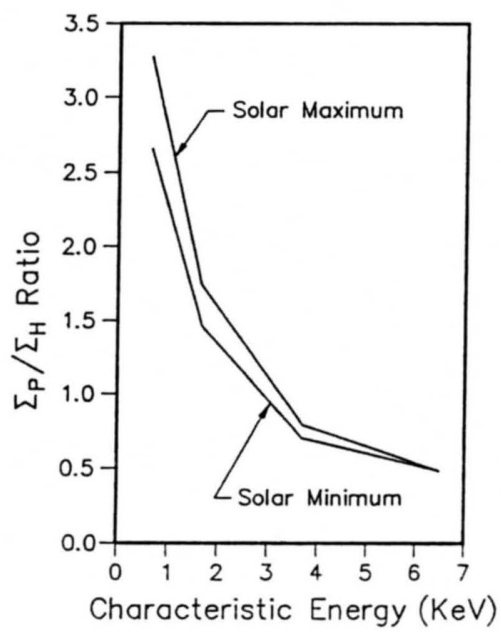

Fig. 9. Ratio of $\Sigma_{P} / \Sigma_{H}$ versus characteristic energy of a $1 \mathrm{erg} / \mathrm{cm}^{2} \mathrm{~s}$ electron flux for winter solar maximum and solar minimum conditions. 

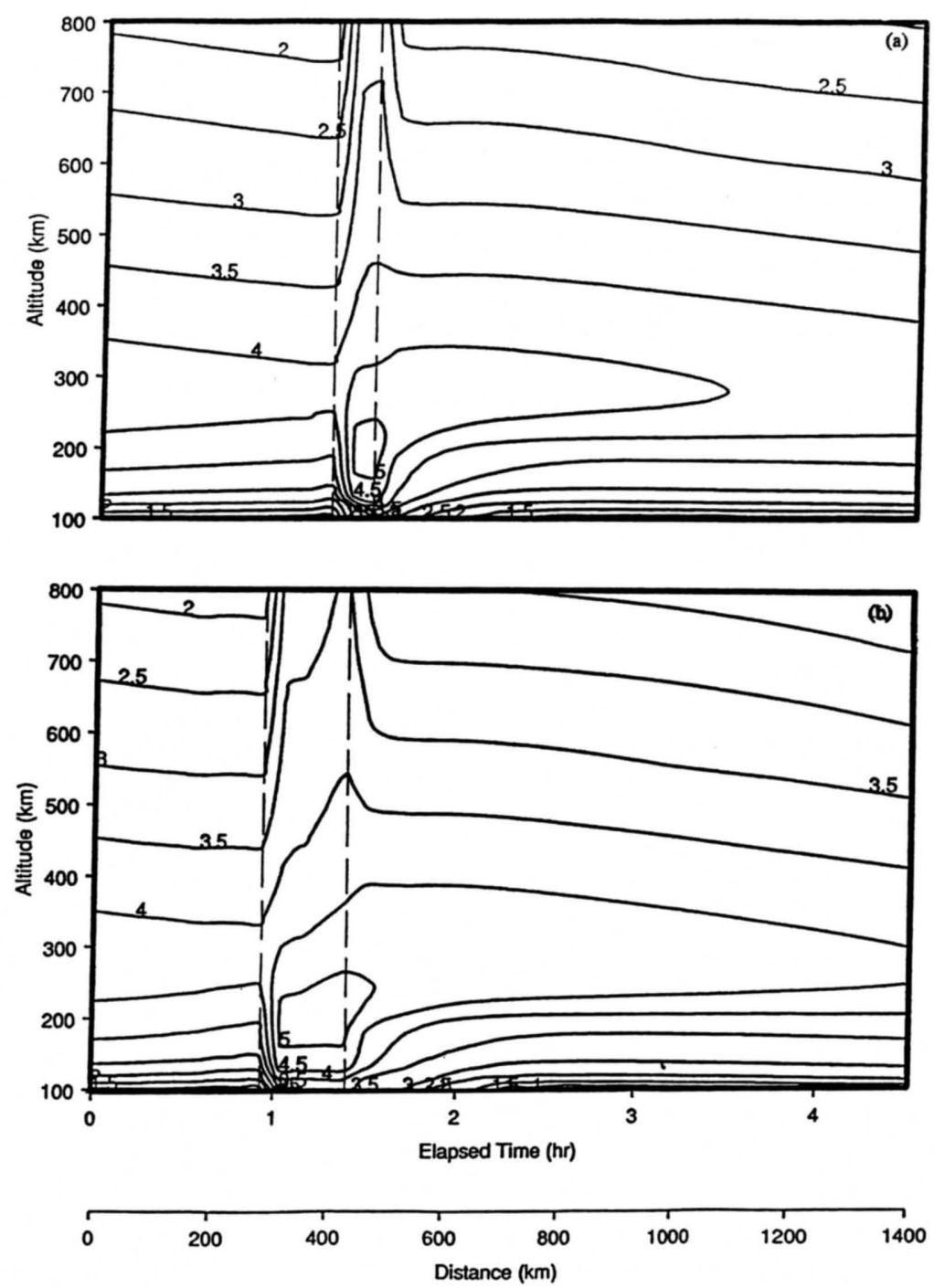

Fig. 10. $N_{e}$ contour cross section of prototype arc with an arc width equal to (a) $100 \mathrm{~km}$ and (b) $400 \mathrm{~km}$. The relevant arc conditions are winter solar minimum, arc electric field equal to $40 \mathrm{mV} / \mathrm{m}$, characteristic energy of precipitating electrons is 0.5 $\mathrm{keV}$, and the energy flux of the precipitation is $1 \mathrm{erg} \mathrm{cm}^{-2} \mathrm{~s}^{-1}$.

These can be compared with Figures $2 a$ and $2 b$ which is for an energy flux of $1 \mathrm{erg} \mathrm{cm}^{-2} \mathrm{~s}^{-1}$.

The most dramatic effect of increasing the flux is seen in the $F$ region. TEC increases by a relative factor of 10 and 50 ,

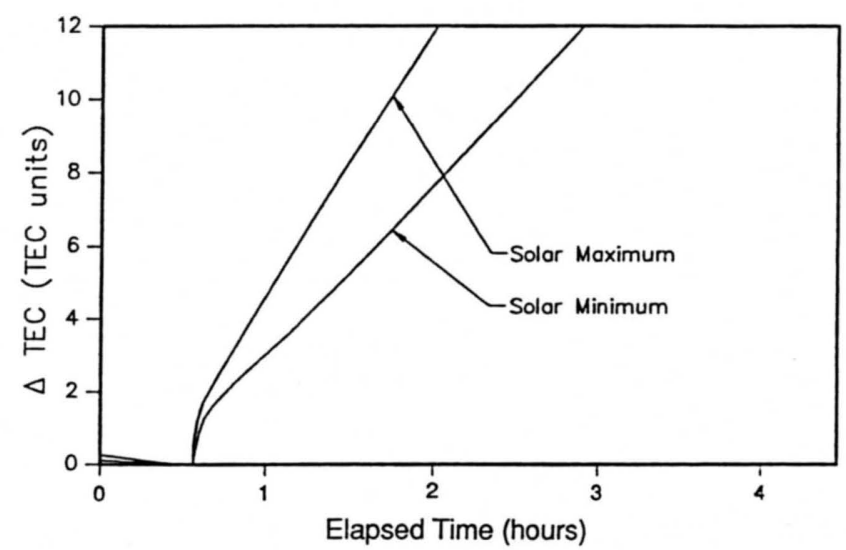

Fig. 11. Delta TEC enhancement due to a $0.5 \mathrm{keV}$ electron flux of 1 $\mathrm{erg} / \mathrm{cm}^{2} \mathrm{~s}$ versus elapsed time in the precipitation region for winter solar maximum $\left(F_{10.7}=215\right)$ and solar minimum conditions $\left(F_{10.7}=75\right)$. Precipitation begins at $\sim 0.5$ hours. respectively, as the flux increases from 0.1 to $1 \mathrm{erg} \mathrm{cm}^{-2} \mathrm{~s}^{-1}$ and then from 0.1 to $5 \mathrm{erg} \mathrm{cm}^{-2} \mathrm{~s}^{-1}$. This nearly linear increase is associated with the almost linear dependence of $F$ region $\mathrm{O}^{+}$chemistry. In contrast, the $E$ region chemistry yields a square root dependence of the electron density upon the ionization flux. This differential $E$ and $F$ region density dependence upon the flux leads to the $\Sigma_{H}$ and $\Sigma_{P}$ having different flux dependences. Figures 2 and 13 show these $\Sigma_{H} / \Sigma_{P}$ differences. For the $0.1 \mathrm{erg} \mathrm{cm}^{-2} \mathrm{~s}^{-1}$ case, the $\Sigma_{H} / \Sigma_{P}$ ratio is 0.5 which decreases to $\sim 0.3$ at $1 \mathrm{erg} \mathrm{cm}^{-2} \mathrm{~s}^{-1}$ and to $\sim 0.25$ at $5 \mathrm{erg} \mathrm{cm}-2 \mathrm{~s}^{-1}$. This specific dependence is also very dependent upon the characteristic energy. In the example shown a $0.5-\mathrm{keV}$ characteristic energy was used. As this increases, less of the ionization occurs in the $F$ region (see preceding section). Consequently, the effect becomes less significant since the $F$ region contribution to $\Sigma_{P}$ is smaller.

\section{Discussion}

In this study we have examined the time dependent modification of the flux tube plasma distribution convecting through an idealized SA arc structure in the midnight (MLT) 


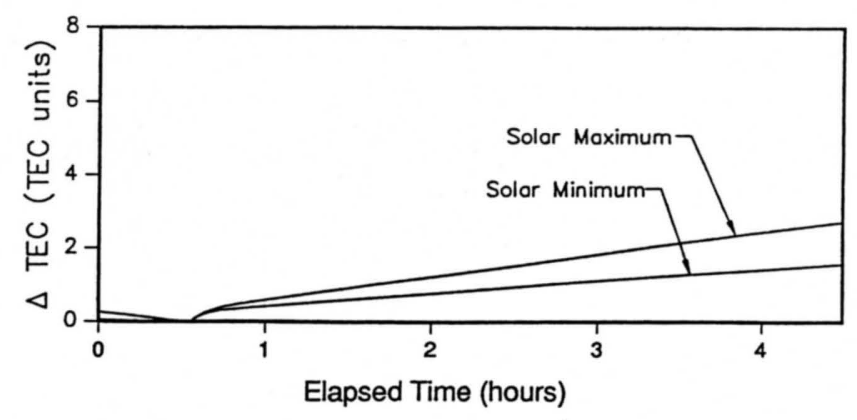

Fig. 12. Same as Figure 11 but for a $0.1 \mathrm{erg} / \mathrm{cm}^{2} \mathrm{~s}$ electron flux.

sector. It is apparent that SA polar cap arcs produced by low characteristic energy precipitation can be a significant source of $F$ region plasma enhancement in nonsunlit portions of the polar cap. Such enhancements and associated density gradients may explain some of the high-latitude scintillation data [Weber and Buchau, 1981; Klobuchar et al., 1985] which are often observed in the winter polar cap in the absence of visible arcs. Additionally, the relative TEC enhancements associated within the SA arcs are consistent with the observations of Basu et al. [1990]. Larger TEC enhancements are seen at solar maximum for the same arc inputs. The reason for this is that the solar maximum $F$ region slab thickness is larger and the peak density occurs at a higher altitude, both of these effects are due primarily to the MSIS neutral profiles assumed for solar maximum and solar minimum. The overall trend is that SA arcs in the solar minimum polar cap produce larger relative TEC enhancements but SA arcs in the solar maximum polar cap produce larger absolute TEC enhancements. This, in turn, implies that the high-altitude electron density gradients should have a solar cycle dependence, being largest during solar minimum.

The parametric study has covered both ionospheric conditions of solar cycle as well as arc parameters (width, energy flux, characteristic energy, and electric field). Although general trends for each have been identified, these trends are heavily coupled to the other parameters. For commonly found arc conditions, widths less than or equal to few hundred

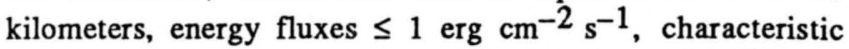
energies $\leq 2 \mathrm{keV}$, and electric field strengths $\leq 40 \mathrm{mV} / \mathrm{m}$ the ionosphere has the same order of magnitude dependence for each of these parameters. The largest $F$ region enhancements are observed in the model for the lowest characteristic energies and moderate $(40 \mathrm{mV} / \mathrm{m})$ electric fields within the arc for winter solar minimum conditions. If the SA arc is sunlit either in a summer hemisphere or in the sunlit noon sector of a winter hemisphere, there is very little $F$ region enhancement. Solar maximum winter conditions lie between these two extreme types of conditions. The polar cap $E$ region enhancements are

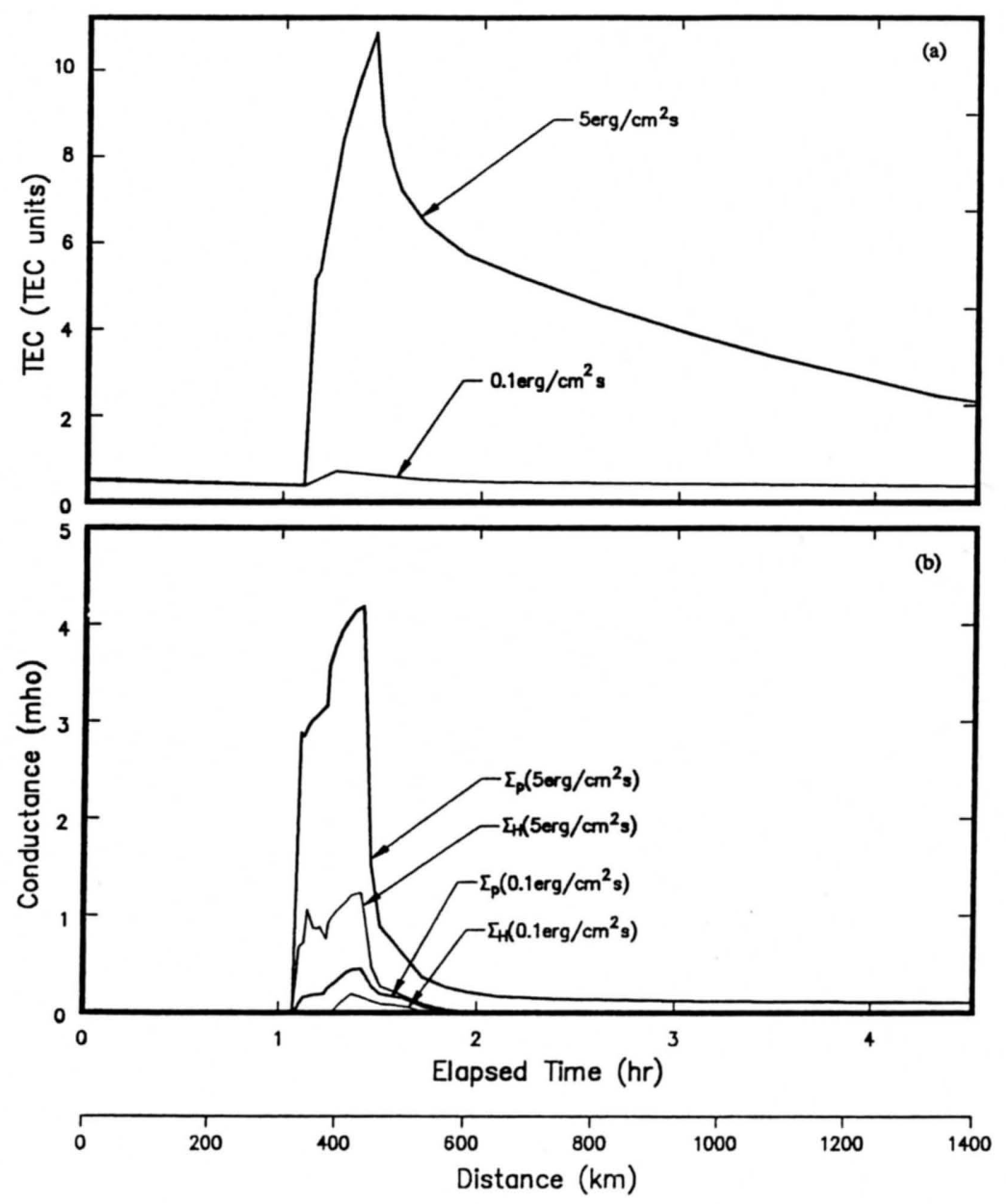

Fig. 13. Variation of TEC, $\Sigma_{H}$ and $\Sigma_{P}$ across the prototype arc with a precipitating energy flux of $0.1 \mathrm{erg} \mathrm{cm}^{-2} \mathrm{~s}^{-1}$ and $5 \mathrm{ergs}$ $\mathrm{cm}^{-2} \mathrm{~s}^{-1}$. The relevant arc conditions are winter solar minimum, arc electric field equal to $40 \mathrm{mV} / \mathrm{m}$, arc width equal to 200 $\mathrm{km}$, and the characteristic energy of precipitating electrons is $0.5 \mathrm{keV}$. 
relatively independent of the background ionospheric condition. This general $E$ and $F$ region difference can lead to significant $\Sigma_{P}$ and $\Sigma_{H}$ differences along an extended SA arc in cases where the solar terminator crosses the arc. Depending on season and UT when the SA arc is partly sunlit or under solstice conditions for SA arc structures which are conjugate in both hemisphere, these variations of $\Sigma_{P}, \Sigma_{H}$ and $F$ region density along the arc may have an important impact on the selfconsistent arc electrodynamics.

Another parameter of consequence in this $E$ and $F$ region comparison is the cross arc drift. For the $E$ region where chemical equilibrium dominates, convection is unimportant and the densities only depend upon the production and loss characteristics. However, for the $F$ region where transport is significant, the density does depend on cross arc transport. In the extreme case of no cross arc transport, $N_{m} F_{2}$ will asymptotically approach a "steady state" value but TEC would increase to very large values. Comparison of these different observations would place strict limits on the degree of stagnation found in SA arcs. Measurements of TEC enhancements associated with SA arcs may be an important tool for diagnosing such arcs. If the flux and characteristic energy of such arcs are known, then the observed TEC enhancement sets a limit upon how much time the flux tube spends within the precipitation region of the arc. This then is useful in determining the degree of plasma cross flow (if any) associated with SA arcs. A parameter which is difficult to measure directly. Cross flow of the plasma can have basically two manifestations. The first which is explicitly modeled here is for the plasma flow to have a convection component across a stationary (with respect to magnetic coordinates) SA arc structure. The second is for the SA structure to move with respect to the background plasma. This is what most SA arcs do at one time or another, move in a dawnward or duskward direction (C. E. Valladares et al., submitted manuscript, 1993). Both methods have similar local effects upon the plasma but each implies a different magnetospheric topology. The first is merely a superposition of an arc electric field structure and a background convection electric field both fixed or varying with UT. The second implies either an arc structure moving relative to the background convection pattern or (if the arc structure is assumed to exist on an adionic line separating two convection cells) the overall convection pattern (and corresponding cell boundaries) are moving with respect to UT. The ability to definitively answer which mechanism produces cross flow is beyond the scope of this work.

What we have done is correlate an observed parameter, i.e., the $\triangle$ TEC enhancement with the modeled $\triangle$ TEC as a function of cross flow, characteristic energy, etc. In the case of the $\Delta$ TEC enhancement we generally reproduce the solar minimum results but are lower than the observed $\triangle$ TEC for solar maximum. This implies that for solar maximum, flux tubes must spend more time within the arc region to produce the observed $\triangle$ TEC enhancement. Otherwise, the flux must be increased or the characteristic energy decreased if the cross flow is the same for solar minimum and solar maximum.

\section{SUMMARY}

In general, we can summarize our results of the ionospheric modification associated with SA polar cap arcs as follows:

1. At low fluxes $\left(<2\right.$ ergs $\left.\mathrm{cm}^{-2} \mathrm{~s}^{-1}\right)$ and low characteristic energies $(<1 \mathrm{keV})$, SA polar cap arcs have the most pronounced relative effect at $F$ region altitudes in darkness for winter solar minimum conditions. The absolute enhancement in summer solar minimum and winter solar maximum is equivalent to that of winter solar minimum, but the higher ambient densities make the relative enhancement less.

2. The modeled TEC enhancements are comparable to observed enhancements and show a similar solar cycle dependence to the results of Basu et al. [1990].

3. The TEC enhancement associated with a SA arc may be used to indicate the degree of plasma cross flow across the arc.

4. For the prototype arc modeled here: (1) The largest $F$ region enhancements increased with the arc electric field strength up to $40 \mathrm{mV} / \mathrm{m}$. As the arc electric field increases above $40 \mathrm{mV} / \mathrm{m}$, enhanced molecular production due to increased $\mathrm{T}_{i}$ decreases the $F$ region enhancement both inside and then beyond the arc. (2) The lowest characteristic energies for the particle precipitation produce the largest $F$ region enhancements and result in a $\Sigma_{H} / \Sigma_{P}$ value below 0.5 . As energy increases, the $F$ region enhancement decreases and the ratio of $\Sigma_{H} \Sigma_{P}$ rises well above 1. (3) Arc width has little effect upon the variation of $\Sigma_{H}$ and $\Sigma_{P}$ across the arc. The $F$ region enhancement for a particular flux and characteristic energy increases as arc width increases and saturates as the width becomes larger than $200 \mathrm{~km}$.

5. As the flux increases for low characteristic energy precipitation, $\Sigma_{P}$ may increase faster than $\Sigma_{H}$ due to enhanced $F$ region densities. This reduces the $\Sigma_{H} \Sigma_{P}$ ratio below 0.5 with increasing flux.

Acknowledgments. This research was supported by NASA grant NAG5-1484 and AFOSR-90-0026 grant to Utah State University.

The Editor thanks two referees for their assistance in evaluating this paper.

\section{REFERENCES}

Akasofu, S.-I., Midday auroras and polar cap auroras, Geofys. Publikasjoner, 29, 73, 1972.

Akasofu, S.-I., and M. Roederer, Polar cap arcs and the open regions, Planet. Space Sci., 29, 803, 1983.

Akasofu, S.-I, and B. Tsurutani, Unusual auroral features observed on January $10-11,1983$ and their possible relationships to the interplanetary magnetic field, Geophys. Res. Lett., 11, 1086, 1984.

Basu, S., S. Basu, E. J. Weber, and G. J. Bishop, Plasma structuring in the polar cap, J. Geomags. Geoelestr., 42, 763, 1990.

Basu, S., S. Basu, E. J. Weber, and W. R. Coley, Case study of polar cap scintillation modeling using DE 2 irregularity measurements at 800 km, Radio Sci., 23, 545, 1988.

Berkey, F. T., L. L. Cogger, and S. Ismail, Evidence for a correlation between sun-aligned arcs and the interplanetary magnetic field direction, Geophys. Res. Lett., 3, 145, 1976.

Berkey, F. T., and Y. Kamide, On the distribution of global auroras during intervals of magnetospheric quiet, J. Geophys. Res., 81, 4701, 1976.

Carlson, H. C., Jr., V. B. Wickwar, E. J. Weber, J. Buchau, J. G. Moore, and W. Whiting, Plasma characteristics of polar cap F-layer arcs, Geophys. Res. Lett., 11, 895, 1984.

Frank L. A., J. D. Craven, J. L. Burch, and J. D. Winningham, Polar views of the earth's aurora with Dynamic Explorer, Geophys. Res. Lett., 9, 1001, 1982.

Frank, L. A., J. D. Craven, D. A. Gurnett, S. D. Shawhan, D. R. Weimer, J. L. Burch, J. D. Winningham, C. R. Chappell, J. H. Waite, R. A. Heelis, N. C. Maynard, M. Sugiura, W. K. Peterson, and E. G. Shelley, The theta aurora, J. Geophys. Res., 91, 3177, 1986.

Gomey, D. J., D. S. Evans, M. S. Gussenhoven, and P. F. Mizera, A multiple-satellite observation of the high-latitude auroral activity on January 11, 1983, J. Geophys. Res., 91, 339, 1986.

Gussenhoven, M. S., Extremely high latitude auroras, J. Geophys. Res., $87,2401-2412,1982$. 
Hardy, D. A., W. J. Burke, and M. S. Gussenhoven, DMSP optical and electron measurements in the vicinity of polar cap arcs, J. Geophys. Res., 87, 2413, 1982.

Hoffman, R. A., R. A. Heelis, J. S. Prasad, A sun-aligned arc observed by DMSP and AE-C, J. Geophys. Res., 90, 9697, 1985.

Ismail, S., D. D. Wallis, and L. L. Cogger, Characteristics of polar cap sun-aligned arcs, J. Geophys. Res., 82, 4741, 1977.

Klobuchar, J. A., G. J. Bishop, and P. H. Doherty, Total electron content and L-band amplitude and phase scintillation measurements in the polar cap ionosphere, AGARD Conf. Proc., 382, 1985.

Lassen, K., and C. Danielson, Quiet time pattem of auroral arcs for different directions of the interplanetary magnetic field in the Y-Z plane, J. Geophys. Res., 83, 5277, 1978

Mende, S. B., J. H. Doolittle, R. M. Robinson, and R. R. Vondrak, Plasma drifts associated with a system of Sun-aligned arcs in the polar cap, $J$. Geophys. Res., 93, 256, 1988.

Mizera, P. F., D. J. Gorney, and D. S. Evans, On the conjugacy of the aurora: High and low latitudes, Geophys. Res. Lett., 14, 190, 1987 ,

Niciejewski, R. J., J. W. Meriwether, Jr., F. G. McCormac, J. H. Hecht, A. B. Christensen, G. G. Sivjee, D. J. Strickland, G. Swenson, S. B. Mende, A. Vallance Jones, R. L. Gattinger, H. C. Carlson, and C. E. Valladares, Coordinated satellite and ground-based measurements of the energy characteristics of a sun-aligned arc over Sondre Stromfjord, J. Geophys. Res., 94, 17,201, 1989.

Obara, T., M. Kitayama, T. Mukai, N. Kaya, J. S. Murphree, and L. L. Cogger, Simultaneous observations of sun-aligned polar cap arcs in both hemispheres by EXOS-C and Viking, Geophys. Res. Lett., 15, $713,1988$.

Petersen, W. K., and E. G. Shelly, Origin of the plasma in a cross-polar cap auroral feature (theta aurora), J. Geophys. Res., 89, 6729, 1984.

Rasmussen, C.E. and R.W. Schunk, A photochemical equilibrium model for ionospheric conductivity, J. Geophys. Res., 93, 9831, 1988.

Schunk, R. W., A mathematical model of the middle and high latitude ionosphere, Pure Appl. Geophys., 127, 255, 1988.

Schunk, R. W., and J. J. Sojka, A theoretical study of the lifetime and transport of large ionospheric density structures, J. Geophys. Res., $92,12,343,1987$.

Schunk, R. W., P. M. Banks, and W. J. Raitt, Effect of electric fields on the daytime high-latitude E- and F-regions, J. Geophys. Res., 80, $3121,1975$.

Schunk, R. W., P. M. Banks, and W. J. Raitt, Effects of electric fields and other processes upon the nighttime high latitude F-layer, J. Geophys. Res., 81, 3271, 1976.

Schunk, R. W., J. J. Sojka, and W. J. Raitt, A comparison of model predictions for plasma convection in the northern and southem polar regions, J. Geophys. Res., 85, 1762, 1980.
Schunk, R. W., J. J. Sojka, and M. D. Bowline, Theoretical study of the electron temperature in the high-latitude ionosphere for solar maximum and winter conditions, J. Geophys. Res., 91, 12,041, 1986.

Sojka, J. J., Global scale, physical models of the $F$ region ionosphere, Rev. Geophys., 27, 371, 1989.

Sojka, J. J., and R. W. Schunk, A theoretical study of the high latitude Fregion's response to magnetospheric storm inputs, J. Geophys. Res., $88,2112,1983$.

Sojka, J. J., and R. W. Schunk, Theoretical study of the high-latitude ionosphere's response to multicell convection patterns, J. Geophys. Res., 92, 8733, 1987.

Sojka, J. J., and R. W. Schunk, A model study of how electric field structures affect the polar cap $F$ region, J. Geophys. Res., 93, 884, 1988.

Valladares, C. E., and H. C. Carlson, Jr., The electrodynamic, thermal, and energetic character of intense sun-aligned arcs in the polar cap, J. Geophys. Res., 96, 1379, 1991.

Vickrey, J. F., R. R. Vondrak, and S. J. Matthews, The diumal and latitudinal variation of auroral zone ionospheric conductivity, $J$. Geophys. Res., 86, 65, 1981.

Weber, E. J., and J. Buchau, Polar cap F-layer auroras, Geophys. Res. Lett., 8, 125, 1981.

Weber, E. J., M. C. Kelley, J. O. Ballenthin, S. Basu, H. C. Carlson, J. R. Fleischman, D. A. Hardy, N. C. Maynard, R. F. Pfaff, P. Rodriguez, R. E. Sheehan, and M. Smiddy, Rocket measurements within a polar cap arc: Plasma, particle, and electric circuit parameters, $J$. Geophys. Res., 94, 6692, 1989.

Weber, E. J., J. Vickrey, H. Gallagher, L. Weiss, C. J. Heinselman, R. A. Heelis, and M. C. Kelley, Coordinated radio and optical measurements of stable auroral arcs at the polar cap boundary, $J$. Geophys. Res., 96, 17,847, 1991.

Zhu, L., J. J. Sojka, R. W. Schunk, and D. J. Crain, A time-dependent model of polar cap arcs, J. Geophys. Res., in press, 1992.

D. J. Crain, R. W. Schunk, J. J. Sojka, and L. Zhu, Center for Atmospheric and Space Sciences, Utah State University, Logan, UT 84322-4405.
(Received February 10, 1992; revised November 2, 1992; accepted November 3,1992 .) 